\title{
Randomised, Double-Blind, Placebo-Controlled Study of a Single Dose of an Amylmetacresol/2,4-dichlorobenzyl Alcohol Plus Lidocaine Lozenge or a Hexylresorcinol Lozenge for the Treatment of Acute Sore Throat Due to Upper Respiratory Tract Infection
}

\author{
Damien McNally, ${ }^{1}$ Adrian Shephard, ${ }^{2}$ Emma Field ${ }^{3}$ \\ ${ }^{1}$ Ormeau Health Centre, Belfast, UK; \\ ${ }^{2}$ Professional Relations, Reckitt Benckiser, Slough, UK; \\ ${ }^{3}$ Global Medical Affairs, Reckitt Benckiser Healthcare, Hull, UK.
}

Received, January 31, 2012; Revised, February 24, 2012; Accepted, April 1, 2012; Published, April 2, 2012.

\begin{abstract}
Purpose: Sore throat is a frequent reason for seeking medical care but few prescription options are available. Lozenges are effective in delivering active ingredients to the throat. This study was conducted to determine the analgesic efficacy of two lozenges - one containing amylmetacresol (AMC)/2,4dichlorobenzyl alcohol (DCBA) and lidocaine and one containing hexylresorcinol - versus placebo in patients with acute sore throat due to upper respiratory tract infection (URTI). Methods: This was a multicentre, randomised, double-blind, parallel group, placebo-controlled study. In total, 190 patients were randomised 1:1:1 to a single dose of AMC/DCBA + lidocaine, hexylresorcinol or placebo lozenge. Subjective ratings of throat soreness, difficulty swallowing, swollen throat, numbing, and sore throat relief were obtained up to 2 hours post dose. Patient and investigator global ratings and a consumer questionnaire were also collected. The primary endpoint was the change from baseline in severity of throat soreness for both lozenges versus placebo at 2 hours post dose. Results: The hexylresorcinol lozenge demonstrated superiority over placebo for primary and secondary efficacy variables including those related to throat soreness, sore throat relief and difficulty swallowing; the AMC/DCBA + lidocaine lozenge was also superior to placebo for secondary endpoints at various time points but did not reach significance for the primary efficacy variable. Both lozenges had a rapid onset of action from 1-10 minutes post dose for the AMC/DCBA + lidocaine lozenge and 1-5 minutes post dose for the hexylresorcinol lozenge. Numbness was reported from 1 minute post dose with the AMC/DCBA + lidocaine lozenge and was greatest at 15 minutes. Numbness was reported from 5 minutes post dose with the hexylresorcinol lozenge and was greatest at 10 minutes. Both lozenges were well tolerated. Conclusions: Both AMC/DCBA + lidocaine and hexylresorcinol lozenges provided rapid and effective sore throat relief in patients with URTI.
\end{abstract}

This article is open to POST-PUBLICATION REVIEW. Registered readers (see "For Readers") may comment by clicking on ABSTRACT on the issue's contents page.

\section{INTRODUCTION}

Sore throat is common and a frequent reason for seeking medical care $(1,2)$. Most sore throats are caused by viruses, in particular rhinoviruses and adenoviruses (3), making antibiotics an inappropriate and ineffective treatment option in most cases $(4,5)$. Indeed, antibiotics are not recommended for the primary treatment of sore throats (6). With few alternative prescription treatment options available, over-the-counter, non-prescription treatments are an important and widely used option for providing relief from symptoms.

Lozenges are an effective format for delivery of active ingredients to the throat. Compared with other delivery formats such as sprays and gargles, lozenges have a long residence time in the mouth (7); this provides for sustained delivery of the active ingredients to the affected site (7).

Lozenges containing the active ingredients amylmetacresol (AMC) and 2,4-dichlorobenzyl alcohol (DCBA) are widely available over-thecounter and have been shown to be highly effective for symptomatic relief of acute sore throat $(8,9)$ and postoperative sore throat $(10)$. A lozenge containing lidocaine hydrochloride, in addition to AMC/DCBA, has also been developed (Strepsils Plus, Reckitt Benckiser, UK).

Corresponding Author: Dr Damien McNally, Ormeau Health Centre, 120 Ormeau Road, Belfast, UK; Email: damienmcnally@doctors.org.uk 
Lidocaine is a well-established local anaesthetic characterised by a rapid onset and intermediate duration of action; it has been previously shown to relieve symptoms of acute pharyngitis when delivered in a lozenge format $(11,12)$. The antiseptic and local anaesthetic hexylresorcinol (13) has also been included as an active ingredient in throat lozenges (Strepsils Extra, Reckitt Benckiser, UK).

A recent questionnaire survey on the use of throat lozenge formulations in healthy volunteers confirmed the anaesthetic effect of AMC/DCBA lozenges, in particular, lozenges containing lidocaine and hexylresorcinol. Higher concentrations of the active ingredients resulted in a greater numbing effect with lozenges that contained hexylresorcinol and lidocaine (14).

This article describes the first randomised study comparing the analgesic efficacy of two lozenges - one containing AMC/DCBA and lidocaine and one containing hexylresorcinol versus placebo in patients with acute sore throat due to an upper respiratory tract infection (URTI). The aim was to characterise the speed and duration of analgesic efficacy of the AMC/DCBA + lidocaine lozenges and hexylresorcinol lozenges, compared with a placebo lozenge in this patient population.

\section{PATIENTS AND METHODS}

\section{Study design}

This was a multicentre, randomised, double-blind, parallel group, placebo-controlled study of a single dose of AMC/DCBA + lidocaine lozenge (Strepsils Plus lozenge, Reckitt Benckiser, UK) or hexylresorcinol lozenge (Strepsils Extra Blackcurrant lozenge, Reckitt Benckiser, UK) versus placebo lozenge for sore throat. The study was conducted between 2 February 2011 and 31 March 2011. Baseline measurements were taken and patients were assessed at intervals up to 120 minutes post dose. After this, patients left the site but recorded any concomitant medication or adverse events up to 24 hours post dose on a diary card and received a follow-up telephone call 1-3 days later.

This study was conducted in accordance with International Conference on Harmonisation Good Clinical Practice and the ethical principles contained within the Declaration of Helsinki (South Africa 1996), as referenced in EU Directive 2001/20/EC.

\section{Patient selection}

Patients with a sore throat ( $\geq 6$ on an 11-point throat soreness scale) due to URTI were screened at eight primary care investigative centres in Northern Ireland after either presenting opportunistically or responding to advertisements in local doctors' surgeries, pharmacies, and some local newspapers. All patients provided written informed consent prior to the study.

Adults (male or female, aged 18-75 years inclusive) were included if they had a sore throat with onset within the previous 4 days due to URTI. Patients had to have at least one out of 40 symptoms of URTI on the URTI questionnaire, and baseline scores of $\geq 6$ on the throat soreness scale, $>50 \mathrm{~mm}$ on the difficulty swallowing scale, and $>33 \mathrm{~mm}$ on the swollen throat scale. Objective confirmation by a physician for the presence of tonsillopharyngitis was required, with a tonsillopharyngitis assessment (TPA) score of $\geq 5$ on a 21 -point scale $(15,16)$.

Exclusion criteria included a sore throat that had been present for more than 4 days, evidence of mouth breathing or severe coughing, existence of other distracting pain (such as mouth ulcer), or concomitant disease with the potential to compromise breathing (for example asthma, bronchopneumonia). Patients were also excluded if they had used any sore throat medication containing a local anaesthetic within the previous 4 hours; analgesic, antipyretic, or cold medication (such as decongestant, antihistamine, antitussive or throat lozenge/spray) within the previous 8 hours; a longer acting or slow-release analgesic during the previous 24 hours (for example piroxicam, naproxen); any medicated confectionary, throat pastille, spray, or any product with demulcent properties (such as boiled sweets) in the previous 2 hours; or antibiotics in the previous 14 days. Women of childbearing potential who were not taking contraceptive precautions, or who were pregnant or lactating, were excluded, as were people with known allergy to the active ingredients or intolerance to fructose. No food, drink, or smoking were allowed during the 2-hour assessment period.

\section{Treatment}

Patients were randomly allocated to one of three groups. Randomisation was achieved by a computer-produced randomisation of study treatments, with subsequent distribution to patients following allocation of a unique number in numerical sequence. 
The study treatments comprised a single dose consisting of one lozenge. Group 1 received one lozenge containing AMC $0.6 \mathrm{mg}, 2,4-\mathrm{DCBA} 1.2$ $\mathrm{mg}$ and lidocaine hydrochloride $10 \mathrm{mg}$; Group 2 received one lozenge containing hexylresorcinol $2.4 \mathrm{mg}$; and Group 3 received a non-medicated sugar-based placebo lozenge.

Double-blinding was achieved by blindfolding the patient before administration of the study treatment by an independent member the clinic staff (who was not involved with any other study-related procedures pre or post dosing). The lozenges were also matched for size. Patients and investigators were therefore unaware of which lozenge the patient had received.

The patients were instructed to suck the lozenge slowly and move it around the mouth without chewing or crunching, until it dissolved. The clinic staff observed the patient to ensure compliance and the mouth was checked to ensure the full dose was consumed.

\section{Efficacy endpoints}

Subjective assessments of analgesic efficacy were made using standard, widely used, published, and reliable methodologies (16-21); including visual analogue scales (VAS) (Figure 1), ordinal, and categorical scales. Multiple assessments were made: these were within 1 minute before the dose (baseline, designated 0 minutes); within \pm 1 minutes of $1,5,10$, and 15 minutes after the dose; and within \pm 5 minutes of $30,45,60,75,90,105$, and 120 minutes after the dose.

The primary efficacy endpoint was the change from baseline at 2 hours in throat soreness. Patients were asked to swallow and circle on an 11-point ordinal throat soreness scale how sore their throat was $(0$ being not sore and 10 being very sore), at baseline and at 2 hours post dose. Secondary endpoints included change from baseline in throat soreness at other time points (1, $5,10,15,30,45,60,75,90$, and 105 minutes post dose) and area under curve (AUC 0-120 minutes). Other secondary endpoints included difficulty swallowing scale (VAS, from not difficult $[0 \mathrm{~mm}]$ to very difficult $[100 \mathrm{~mm}]$ ), swollen throat scale (VAS, from not swollen [0 $\mathrm{mm}]$ to very swollen $[100 \mathrm{~mm}])$, throat numbness scale (5-point categorical scale: none, mild, moderate, considerable, complete numbness), and sore throat relief scale (7-point categorical scale: no relief; or slight, mild, moderate, considerable, almost complete, or complete relief). These were all assessed as change from baseline at 1, 5, 10,
$15,30,45,60,75,90,105$, and 120 minutes post dose and AUC 0-120 minutes.

Onset of analgesia was defined as the time to first reporting 'moderate pain relief' which was the mid-point on the 7-point sore throat relief scale. At 2 hours post dose, patients were asked to complete a grading for the study treatment using a standard 5-point category scale (Patient's Global Evaluation of the Study Medication as a Treatment of Sore Throat) and the investigator was similarly asked to rate the patient's response to the study treatment (Practitioner's Clinical Assessment of the Study Medication as a Treatment of Sore Throat). Patients also completed a consumer questionnaire at this time.

\section{Safety endpoints}

Safety and tolerability of the lozenges were assessed in terms of the overall proportion of patients who reported adverse events and serious adverse events during the 2 hours of observation and in the 24-hour period following administration of the lozenge.

\section{STATISTICAL ANALYSIS}

Sample size calculations were based on a previous study (22). This found that the difference in the change in throat soreness from baseline at 2 hours between an AMC/DCBA lozenge and placebo for patients with a TPA score $\geq 5$ and a score of at least 6 on the 11-point throat soreness scale was -1.21 with a pooled standard deviation (SD) of 1.78. Assuming similar variability for the current study, 57 patients per arm would be sufficient to provide $95 \%$ power to detect a difference of -1.21 in the mean change from baseline in severity of throat soreness using a 2-sample t-test at the $5 \%$ significance level. In order to account for dropouts, recruitment of a total of 190 patients was set.

The primary efficacy endpoint was analysed by analysis of covariance (ANCOVA) with baseline throat soreness severity as a covariate and factors for treatment group and centre.

Treatment group differences were estimated using the mean square error from the ANCOVA and using Fisher's protected least significant difference (LSD) method (if the overall treatment effect in the ANCOVA model was significant at the $5 \%$ level, the comparisons of the AMC/DCBA + lidocaine and hexylresorcinol lozenge groups versus placebo lozenge group were performed without any requirement to adjust the significance level for the pairwise comparisons). 
Throat Soreness Scale (0-10 ordinal scale)

Circle the number on the scale that shows how sore your throat is when you swallow

(not sore) $\begin{array}{lllllllllll}1 & 2 & 3 & 4 & 5 & 6 & 7 & 8 & 9 & 10 \text { (very sore) }\end{array}$

Difficulty Swallowing Scale (100 $\mathrm{mm}$ visual analogue scale)

Place a line through the scale that shows how difficult it is to swallow

$\begin{array}{ll}\text { (not difficult) } 100 \mathrm{~mm} & \\ 0 \mathrm{~mm} & 100 \text { difficult) }\end{array}$

Swollen Throat Scale (100 mm visual analogue scale)

Place a line through the scale that shows how swollen your throat feels now $\begin{array}{lll}\text { (not swollen) } 10 \mathrm{~mm} & \\ 0 \mathrm{~mm} & 100 \mathrm{~mm}\end{array}$

Throat Numbness Scale

(5-point categorical scale)

None

Mild

Moderate

Considerable

Complete numbness
Sore Throat Relief Scale

(7-point categorical scale)

No relief

Slight

Mild

Moderate

Considerable

Almost complete

Complete relief

Figure 1. Subjective Assessment Scales for Throat Soreness, Difficulty Swallowing and Swollen Throat

The $95 \%$ confidence interval for the difference in least square means was estimated using the mean square error from the ANCOVA.

All secondary endpoints were analysed without any procedures to account for multiple comparisons. The ANCOVA model was also used for secondary throat soreness measures (AUC and change from baseline to 2 hours), and for throat numbness and sore throat relief (AUC and change from baseline), as well as the Patient's Global Evaluation of the Study Medication as a Treatment of Sore Throat and the Practitioner's Clinical Assessment of the Study Medication as a Treatment of Sore Throat. Similarly, ANCOVA (with factors for treatment group and centre and covariates for baseline throat soreness) was used for difficulty swallowing and swollen throat, with an additional covariate to account for the respective baseline measure.

Onset of analgesia was compared between treatment groups and placebo using a Cox proportional hazards model with factors for treatment group and centre and a covariate for baseline throat soreness severity. Patients not reporting at least moderate sore throat relief were censored at the time of their last recorded followup assessment.

For the consumer questionnaire, questions on non-numeric ordinal scales were analysed using a proportional odds model with factors for treatment group and centre and a covariate for baseline throat soreness. Questions on numeric ordinal scales were analysed using the same ANCOVA model as the primary efficacy endpoint. Exceptions were 'How much do you feel like your best overall?' and 'How happy are you, in relation to your throat?' which included covariates for the relevant baseline score for the specific question.

Data are presented as mean \pm SD. All analyses were conducted using SAS Version 9.2. All statistical tests were 2-tailed with $\mathrm{p}<0.05$ considered significant. The null hypothesis at all times was the equality of the treatments being compared. The normality of data distribution was checked by an examination of the residual plots and the Shapiro-Wilk test of normality. All the AUC analyses were based on actual rather than 
scheduled timings and calculated using the trapezoidal rule, with last observation carried forward.

The full analysis set consisted of all patients who were randomised and took the study medication. The safety set comprised all patients who took the study medication (and was identical to the full analysis set in this trial). The perprotocol set comprised a subset of the full analysis set and consisted of all patients who satisfied all of the inclusion/exclusion criteria, who correctly received the treatment to which they were randomised, and who successfully completed the study up to the 2-hour assessment.

\section{RESULTS}

\section{Patient disposition}

A total of 190 patients were randomised: 64 patients to the AMC/DCBA + lidocaine lozenge group, 64 patients to the hexylresorcinol lozenge group, and 62 patients to the placebo lozenge group. All patients completed the study.

A total of 16 patients had protocol deviations, including some with more than one deviation: six patients (3.2\%) reported difficulty swallowing $\leq 50 \mathrm{~mm}$ at screening; five patients $(2.6 \%)$ reported swollen throat $\leq 33 \mathrm{~mm}$ at screening; and four patients $(2.1 \%)$ had a throat soreness score $\leq 6$ at baseline. Five $(2.6 \%)$ patients reported no symptoms on the URTI questionnaire at baseline, and a further patient had assessments not performed within the admissible scheduled time interval. These patients were excluded from the per-protocol analysis set.

\section{Patient demographics}

The treatment groups were matched for demographic variables with the age of the patients ranging from 18 to 73 with a mean of 31.6 years (Table 1). The placebo group contained more males (58\%) compared with each of the other two groups (33\%) (Table 1). The mean duration of URTI was 2.11 days and mean duration of sore throat was 2.12 days (Table 1 ).

The mean TPA score at screening was 8.4 (range 5 to 17). The majority of patients (73\%) had moderate-severe sore throat as assessed by the practitioner: 16 patients (8\%) had severe inflammation, 124 (65\%) had moderate inflammation, and 48 (25\%) had mild inflammation.

\begin{tabular}{|c|c|c|c|c|}
\hline & $\begin{array}{l}\mathrm{AMC} / \mathrm{DCBA}+ \\
\text { lidocaine } \\
\text { lozenge }\end{array}$ & $\begin{array}{l}\text { Hexyl- } \\
\text { resorcinol } \\
\text { lozenge }\end{array}$ & Placebo & Overall \\
\hline Number of patients & 64 & 64 & 62 & 190 \\
\hline Age (years), mean \pm SD & $32.4 \pm 15.8$ & $30.9 \pm 12.8$ & $31.5 \pm 11.7$ & $31.6 \pm 13.5$ \\
\hline Sex, $\%$ male & 32.8 & 32.8 & 58.1 & 41.1 \\
\hline Race, \% Caucasian & 100 & 98.4 & 95.2 & 97.9 \\
\hline Alcohol drinker, $\%$ & 68.8 & 71.9 & 74.2 & 71.6 \\
\hline Current smoker, $\%$ & 29.7 & 32.8 & 32.3 & 31.6 \\
\hline Former smoker, $\%$ & 14.1 & 12.5 & 11.3 & 12.6 \\
\hline $\begin{array}{l}\text { Duration of sore throat } \\
\text { (days), mean } \pm \text { SD }\end{array}$ & $2.17 \pm 0.90$ & $1.98 \pm 0.92$ & $2.19 \pm 0.87$ & $2.12 \pm 0.90$ \\
\hline $\begin{array}{l}\text { Duration of URTI (days), } \\
\text { mean } \pm \text { SD }\end{array}$ & $2.14 \pm 0.83$ & $2.02 \pm 0.92$ & $2.16 \pm 0.91$ & $2.11 \pm 0.88$ \\
\hline $\begin{array}{l}\text { Throat soreness score }{ }^{1}, \\
\text { mean } \pm \text { SD }\end{array}$ & $7.20 \pm 1.12$ & $7.27 \pm 1.21$ & $7.13 \pm 1.00$ & $7.20 \pm 1.11$ \\
\hline $\begin{array}{l}\text { Difficulty swallowing } \\
\text { score }^{2}, \text { mean } \pm \text { SD }\end{array}$ & $72.5 \pm 10.5$ & $73.6 \pm 12.1$ & $70.8 \pm 11.9$ & $72.3 \pm 11.5$ \\
\hline $\begin{array}{l}\text { Throat swollen score }{ }^{3}, \\
\text { mean } \pm \text { SD }\end{array}$ & $66.1 \pm 16.3$ & $68.3 \pm 18.1$ & $66.7 \pm 15.2$ & $67.1 \pm 16.5$ \\
\hline \multicolumn{5}{|c|}{$\begin{array}{l}{ }^{1} \text { Assessment of throat soreness on a } 11 \text {-point scale }(0=\text { not sore and } 10=\text { very sore }) \\
{ }^{2} \text { VAS of difficulty swallowing }(0 \mathrm{~mm}=\text { not difficult, } 100 \mathrm{~mm}=\text { very difficult }) \\
{ }^{3} \text { VAS of swollen throat }(0 \mathrm{~mm}=\text { not swollen, } 100 \mathrm{~mm}=\text { very swollen })\end{array}$} \\
\hline
\end{tabular}


The most common URTI symptoms patients reported experiencing immediately prior to the dose of study medication were sore throat (179 patients, 94\%), cough (96 patients, 51\%), and throat tickle ( 89 patients, $47 \%$ ). Overall, the mean number of URTI symptoms reported was 7.0 with a maximum of 24 (out of 40 on the questionnaire). The mean number of URTI symptoms was 7.0 in the AMC/DCBA + lidocaine lozenge group, 7.5 in the hexylresorcinol lozenge group, and 6.7 in the placebo group.

Concomitant medication use varied slightly between groups, with 36 patients $(56 \%)$ in the AMC/DCBA + lidocaine lozenge group, 31 patients $(48 \%)$ in the hexylresorcinol lozenge group and 26 patients (42\%) in the placebo group taking medications at the time of randomisation. These included one patient in the AMC/DCBA + lidocaine lozenge group who was taking the antiinflammatory etodolac once daily for rheumatoid arthritis, and three patients in the hexylresorcinol lozenge group who were taking analgesics (one taking acetaminophen three-times daily for sore throat commencing 51.25 hours prior to dosing; one taking acetaminophen as required for foot pain; and one taking tramadol as required for arthralgia) at baseline.

\section{Efficacy results}

\section{Primary endpoint}

The change from baseline to 2 hours was significantly greater than placebo for the hexylresorcinol lozenge $(\mathrm{p}=0.004)$ (Table 2$)$. The pairwise difference between the AMC/DCBA + lidocaine lozenge and placebo approached but did not reach statistical significance $(\mathrm{p}=0.06)$ (Table 2 ). Analysis of the per-protocol set revealed similar results.

\section{Secondary endpoints}

Throat soreness was significantly improved versus placebo for both active lozenges, assessed by AUC 0-120 mins (Table 3) and change from baseline (Figure 2a). Throat soreness scores for the hexylresorcinol lozenge were significantly better than placebo at all time points from 1 minute post dose, while the throat soreness scores for the AMC/DCBA + lidocaine lozenge were significantly better than placebo from 5-30 minutes (Figure 2a). Maximum reductions in throat soreness were recorded at 15 minutes post dose for both active lozenges (Figure 2a).

Difficulty swallowing was significantly improved versus placebo for both active lozenges, assessed by AUC 0-120 mins (Table 3) and change from baseline (Figure 2b). Difficulty swallowing scores for the hexylresorcinol lozenge were significantly better than placebo at all time points from 1 minute post dose, while scores for the $\mathrm{AMC} / \mathrm{DCBA}+$ lidocaine lozenge were significantly better versus placebo from 5 minutes onwards (Figure $2 b$ ).

\begin{tabular}{|c|c|c|c|c|c|}
\hline & $\begin{array}{c}\text { AMC/DCBA + } \\
\text { lidocaine } \\
\text { lozenge }\end{array}$ & $\begin{array}{l}\text { Hexyl-resorcinol } \\
\text { lozenge }\end{array}$ & Placebo & $\begin{array}{c}\text { AMC/DCBA } \\
+ \text { lidocaine } \\
\text { lozenge vs } \\
\text { placebo }\end{array}$ & $\begin{array}{c}\text { Hexyl- } \\
\text { resorcinol } \\
\text { lozenge vs } \\
\text { placebo }\end{array}$ \\
\hline $\mathrm{N}$ & $63^{c}$ & 64 & 62 & & \\
\hline Baseline, mean $\pm \mathrm{SD}$ & $7.16 \pm 1.07$ & $7.27 \pm 1.21$ & $7.13 \pm 1.00$ & & \\
\hline $\begin{array}{c}120 \text { minutes post dose } \\
\text { mean } \pm \text { SD }\end{array}$ & $5.41 \pm 2.34$ & $5.05 \pm 2.62$ & $6.16 \pm 1.87$ & & \\
\hline $\begin{array}{c}\text { Change from baseline, } \\
\text { mean } \pm \text { SD }\end{array}$ & $-1.75 \pm 2.31$ & $-2.22 \pm 2.66$ & $-0.97 \pm 1.96$ & & \\
\hline LS mean ${ }^{a}$ & -1.78 & -2.19 & -1.03 & & \\
\hline Difference in LS mean ${ }^{\mathrm{b}}$ & & & & $\begin{array}{c}-0.75 \\
(95 \% \mathrm{CI} \\
-1.54 \text { to } 0.04) \\
\mathrm{p}=0.06\end{array}$ & $\begin{array}{c}-1.16(95 \% \mathrm{CI} \\
-1.95 \text { to } \\
-0.37) \\
\mathrm{p}=0.004\end{array}$ \\
\hline \multicolumn{6}{|c|}{$\begin{array}{l}\text { LS; least square } \\
{ }^{\mathrm{a}} \text { Estimated from ANCOVA model with factors for treatment and centre and a covariate for baseline throat soreness } \\
\text { bA negative difference favours test lozenge over placebo } \\
{ }^{c} \text { No data available for one patient in the AMC/DCBA + lidocaine lozenge group }\end{array}$} \\
\hline
\end{tabular}


Swollen throat was significantly improved versus placebo for both active lozenges, assessed by AUC 0-120 mins (Table 3) and change from baseline (Figure 2c). Swollen throat scores for the hexylresorcinol lozenge group were significantly better than placebo at all time points from 1 minute post dose, while scores for the AMC/DCBA + lidocaine lozenge group were significantly better versus placebo from 10-45 minutes and from 75-120 minutes (Figure 2c).

Throat numbness was significantly greater versus placebo for both active lozenges, assessed by AUC 0-120 mins (Table 3) and change from baseline (Figure 2d). Throat numbness scores with the hexylresorcinol lozenge were significantly better than placebo at all time points from 5 minutes post dose and were maximal at 10 minutes (Figure 2d). Throat numbness scores with the AMC/DCBA + lidocaine lozenge were significantly better than placebo at all time points from 1 minute post dose, and were maximal at 15 minutes (Figure 2d).

Sore throat relief was significantly better versus placebo for both active lozenges, assessed by AUC (0-120 mins) (Table 3$)$ and change from baseline (Figure 2e). Sore throat relief was significantly better than placebo for both active lozenges at all time points from 1 minute post dose (Figure 2e). Maximum sore throat relief was recorded at 15 minutes post dose for both active lozenges (Figure 2e).

Onset of analgesia (time to moderate pain relief) occurred earlier for the hexylresorcinol lozenge than for the AMC/DCBA + lidocaine lozenge, with Kaplan-Meier medians of 12.5 minutes and 30 minutes, respectively. In total, at least moderate pain relief was reported by $46 / 64$ $(72 \%)$ patients in the hexylresorcinol lozenge group, 39/64 (61\%) in the AMC/DCBA + lidocaine lozenge group and 13/62 (21\%) in the placebo lozenge group. The pairwise comparisons between the two active lozenges and placebo were highly statistically significant $(\mathrm{p}<0.0001)$.

Practitioner's assessment found that both active lozenges were rated highly statistically significantly better than placebo $(\mathrm{p}<0.0001)$ (Table 4). Patient's assessment also found that both active lozenges were rated highly statistically significantly better than placebo $(\mathrm{p}<0.0001)$ (Table 4).

The consumer questionnaire at 2 hours found a statistically significant benefit for both active lozenges over placebo on a range of questions, including how effective the lozenge was $(\mathrm{p}<0.001$ for both lozenges), the depth of numbing
( $<<0.0001$ for both lozenges), intensity of the numbing $(\mathrm{p}<0.0001$ for both lozenges), feeling their best overall $(p=0.002$ for AMC/DCBA + lidocaine lozenge and $\mathrm{p}=0.0003$ for hexylresorcinol lozenge), and how happy they were with their throat $(p<0.0001$ for AMC/DCBA + lidocaine lozenge and $p=0.0001$ for hexylresorcinol lozenge). This significant difference was also reflected in the patients' response to feeling less distracted $(\mathrm{p}=0.0033$ for AMC/DCBA + lidocaine lozenge and $\mathrm{p}=0.0004$ for hexylresorcinol lozenge), making them feel better than before $(\mathrm{p}=0.0002$ for AMC/DCBA + lidocaine lozenge and $\mathrm{p}<0.0001$ for hexylresorcinol lozenge), and taking their mind off the pain ( $<<0.0001$ for both lozenges). Both active lozenges were found to offer highly significant soothing effects over the placebo lozenge $(\mathrm{p}<0.0001)$.

\section{Safety results}

There were no statistically significant differences between groups in the proportion of patients reporting treatment-emergent adverse events. There were a total of seven adverse events reported by six patients. In the hexylresorcinol lozenge group, one patient $(2 \%)$ reported two adverse events. In the AMC/DCBA + lidocaine group, one patient (2\%) reported one adverse event. In the placebo group, four patients $(6 \%)$ reported four adverse events. The majority of events were mild in severity with one event (earache) in the placebo group being classed as severe. None were considered to be definitely, probably, or possibly related to the study medication.

\section{DISCUSSION}

Both the hexylresorcinol lozenge and AMC/DCBA + lidocaine lozenge demonstrated fast and effective relief from the symptoms of acute sore throat due to URTI. The hexylresorcinol lozenge demonstrated superiority over placebo for primary and secondary efficacy variables including those related to throat soreness, sore throat relief and difficulty swallowing; the AMC/DCBA + lidocaine lozenge was also superior to placebo on secondary endpoints at various time points but did not reach significance on the primary efficacy variable. 


\begin{tabular}{|c|c|c|c|c|c|}
\hline & $\begin{array}{l}\text { AMC/DCBA } \\
+\quad \text { lidocaine } \\
\text { lozenge }\end{array}$ & $\begin{array}{l}\text { Hexyl- } \\
\text { resorcinol } \\
\text { lozenge } \\
\end{array}$ & Placebo & $\begin{array}{l}\text { AMC/DCBA }+ \\
\text { lidocaine lozenge vs } \\
\text { placebo }\end{array}$ & $\begin{array}{l}\text { Hexyl-resorcinol } \\
\text { lozenge vs placebo }\end{array}$ \\
\hline \multicolumn{6}{|l|}{ Throat soreness } \\
\hline $\mathrm{N}$ & 64 & 64 & 62 & & \\
\hline Mean \pm SD & $-1.80 \pm 1.84$ & $-2.38 \pm 1.94$ & $-1.09 \pm 1.64$ & & \\
\hline $\mathrm{LS}_{\text {mean }}{ }^{\mathrm{a}}$ & -1.85 & -2.39 & -1.18 & & \\
\hline Difference in LS mean $^{c}$ & & & & $\begin{array}{l}-0.66(95 \% \text { CI }-1.28 \\
\text { to }-0.05) p=0.03\end{array}$ & $\begin{array}{llr}-1.21 & (95 \% & \mathrm{CI} \\
-1.82 & \text { to } & -0.59) \\
\mathrm{p}=0.0001 & \end{array}$ \\
\hline \multicolumn{6}{|l|}{ Difficulty swallowing } \\
\hline $\mathrm{N}$ & 64 & 64 & 62 & & \\
\hline Mean \pm SD & $-19.1 \pm 20.0$ & $-27.3 \pm 21.9$ & $-8.0 \pm 11.6$ & & \\
\hline LS mean ${ }^{b}$ & -19.3 & -27.2 & -8.6 & & \\
\hline Difference in LS mean ${ }^{c}$ & & & & $\begin{array}{l}-10.7(95 \% \text { CI }-17.1 \\
\text { to }-4.3) \mathrm{p}=0.0012\end{array}$ & $\begin{array}{llr}-18.7 & (95 \% & \mathrm{CI} \\
-25.1 & \text { to } & -12.2) \\
\mathrm{p}<0.0001 & \end{array}$ \\
\hline \multicolumn{6}{|l|}{ Swollen Throat } \\
\hline $\mathrm{N}$ & 63 & 64 & 62 & & \\
\hline Mean \pm SD & $-14.4 \pm 19.4$ & $-22.8 \pm 23.3$ & $-5.9 \pm 14.6$ & & \\
\hline LS mean $^{\mathrm{a}}$ & -14.9 & -22.5 & -6.2 & & \\
\hline Difference in LS mean ${ }^{c}$ & & & & $\begin{array}{l}-8.8(95 \% \text { CI }-15.3 \\
\text { to }-2.2) \mathrm{p}=0.009\end{array}$ & $\begin{array}{l}-16.3 \quad(95 \% \text { CI } \\
-22.9 \text { to }-9.8) \\
p<0.0001\end{array}$ \\
\hline \multicolumn{6}{|l|}{ Throat numbness } \\
\hline $\mathrm{N}$ & 64 & 64 & 62 & & \\
\hline Mean \pm SD & $2.13 \pm 0.98$ & $2.30 \pm 0.99$ & $1.64 \pm 0.74$ & & \\
\hline $\mathrm{LS}_{\text {mean }}{ }^{\mathrm{a}}$ & 2.11 & 2.27 & 1.63 & & \\
\hline Difference in LS mean $^{\mathrm{d}}$ & & & & $\begin{array}{l}0.49(95 \% \text { CI } 0.17 \\
\text { to } 0.80) p=0.0024\end{array}$ & $\begin{array}{l}0.64(95 \% \text { CI } 0.33 \\
\text { to } 0.96) \mathrm{p}<0.0001\end{array}$ \\
\hline \multicolumn{6}{|l|}{ Sore throat relief } \\
\hline $\mathrm{N}$ & 64 & 64 & 62 & & \\
\hline Mean \pm SD & $1.86 \pm 1.33$ & $2.28 \pm 1.41$ & $0.81 \pm 0.95$ & & \\
\hline LS mean $^{\mathrm{a}}$ & 1.90 & 2.31 & 0.84 & & \\
\hline Difference in LS mean $^{\mathrm{d}}$ & & & & $\begin{array}{l}1.06(95 \% \text { CI } 0.62 \\
\text { to } 1.50) \mathrm{p}<0.0001\end{array}$ & $\begin{array}{l}1.47(95 \% \text { CI } 1.03 \\
\text { to } 1.91) \mathrm{p}<0.0001 \\
\end{array}$ \\
\hline \multicolumn{6}{|c|}{$\begin{array}{l}{ }^{a} \text { Estimated from ANCOVA model with factors for treatment and centre and a covariate for baseline throat soreness } \\
{ }^{b} \text { Estimated from ANCOVA model with factors for treatment and centre and covariates for baseline throat soreness } \\
\text { and baseline score for difficulty in swallowing } \\
\text { cA negative difference favours test lozenge over placebo } \\
{ }^{d} \text { A positive difference favours test lozenge over placebo }\end{array}$} \\
\hline
\end{tabular}

Significantly greater improvements in severity of throat soreness, difficulty swallowing, swollen throat symptoms, and greater sore throat relief, were reported with both lozenges compared with placebo. In addition, this trial clearly showed the anaesthetic effect of both hexylresorcinol and AMC/DCBA + lidocaine lozenges formulations, as evidenced by the significantly greater numbness scores achieved versus placebo. This supports data in healthy volunteers who reported a concentration-dependent numbing effect with hexylresorcinol or lidocaine-containing lozenges (14).

The hexylresorcinol lozenge demonstrated a rapid onset of action that was evident as early as 1-5 minutes after the dose. The maximal effect was evident on most efficacy outcomes at 15 minutes, with onset of moderate pain relief at 12.5 minutes. Numbness was reported from 5 minutes post dose, and was maximal at 10 minutes. 
a

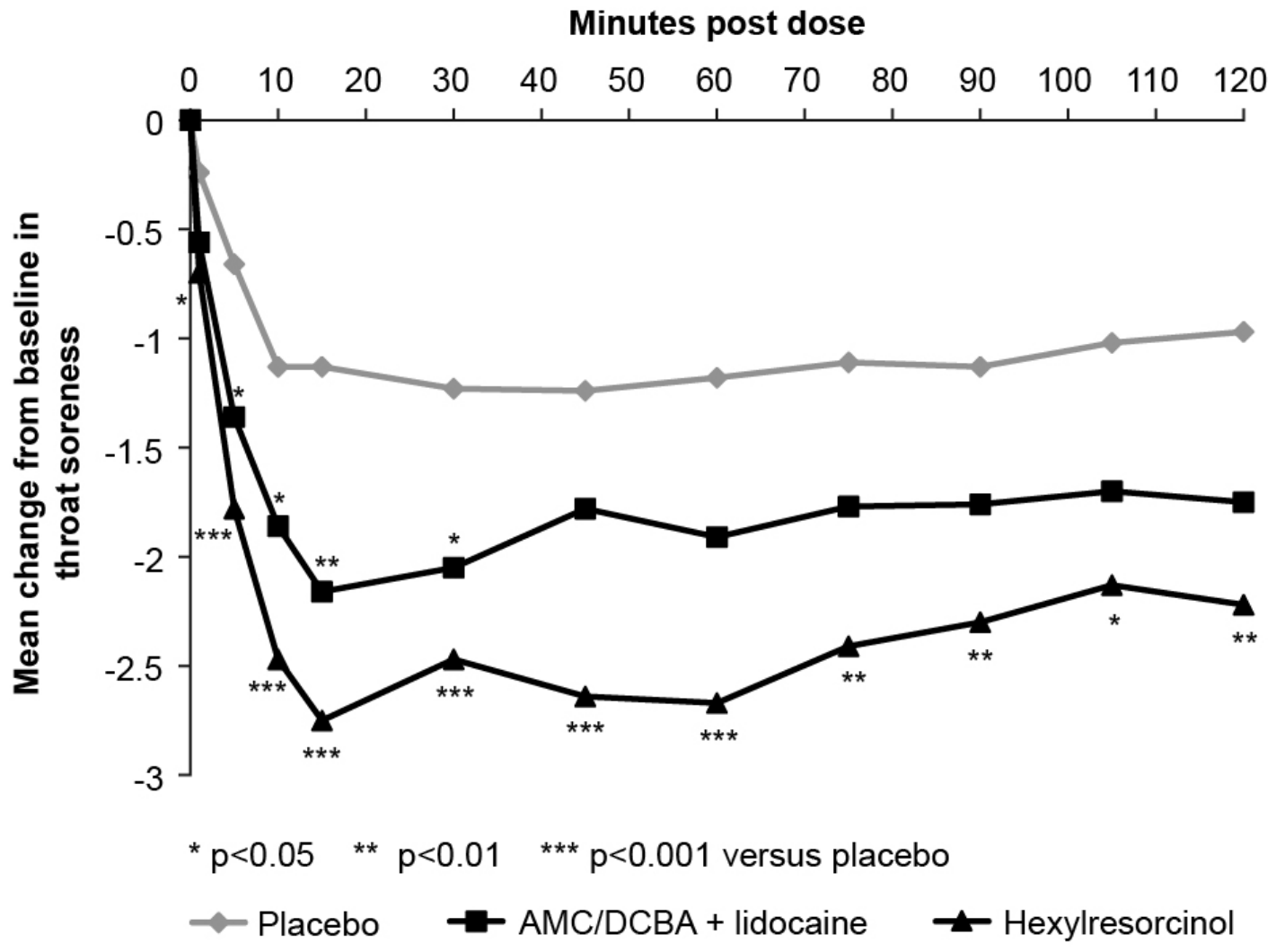

b

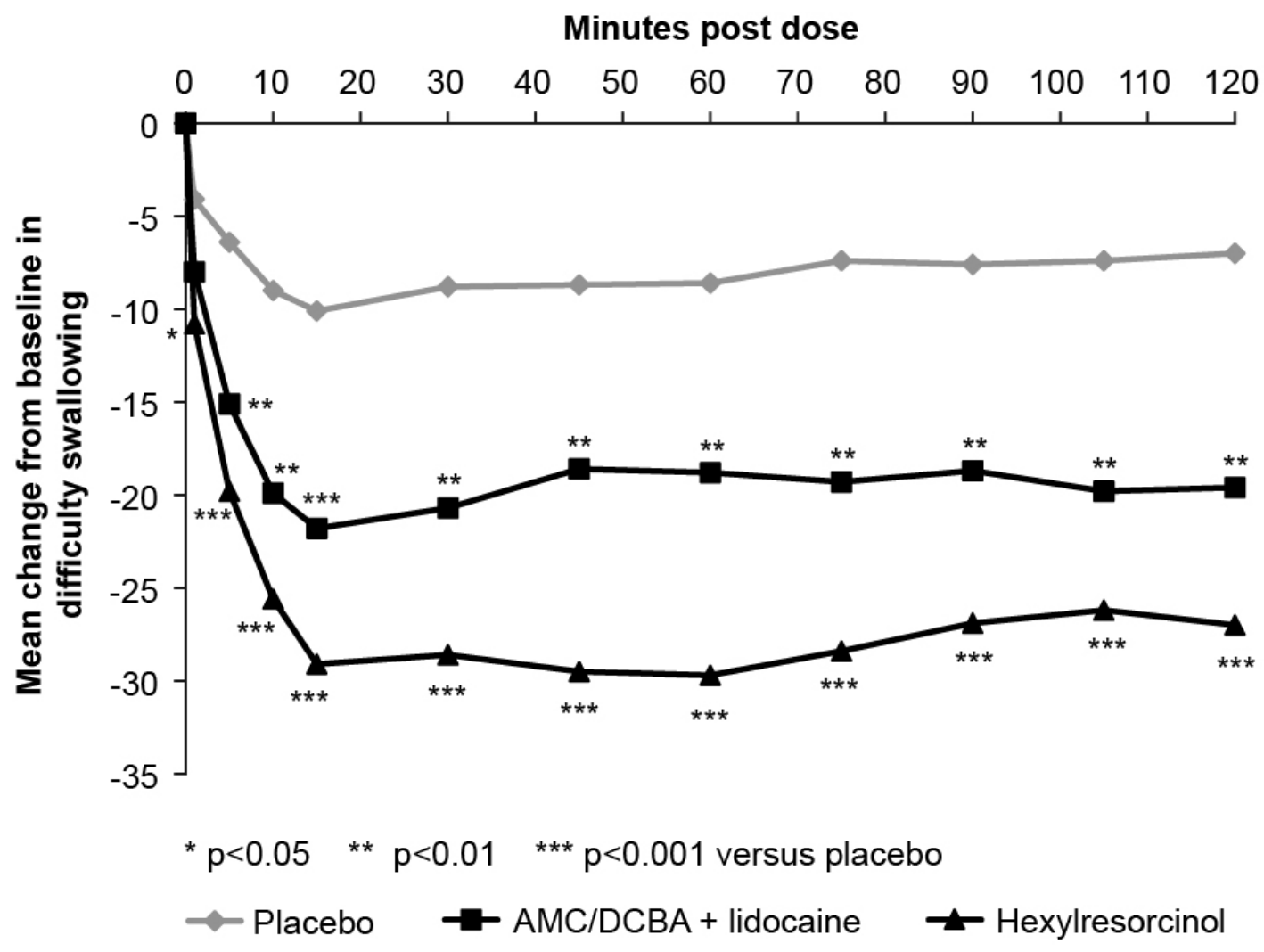


Figure 2 Continued.....

c

Minutes post dose

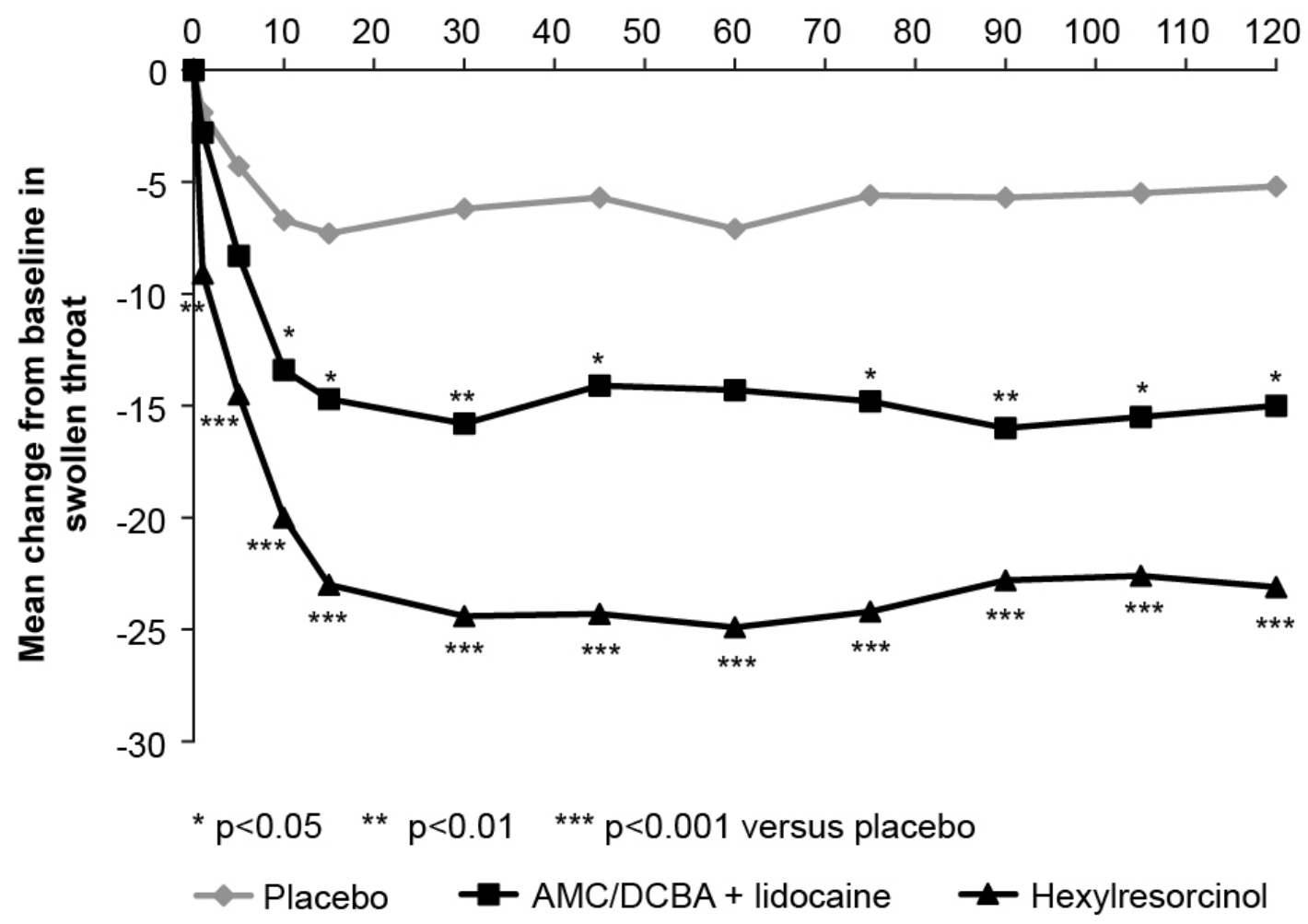

d

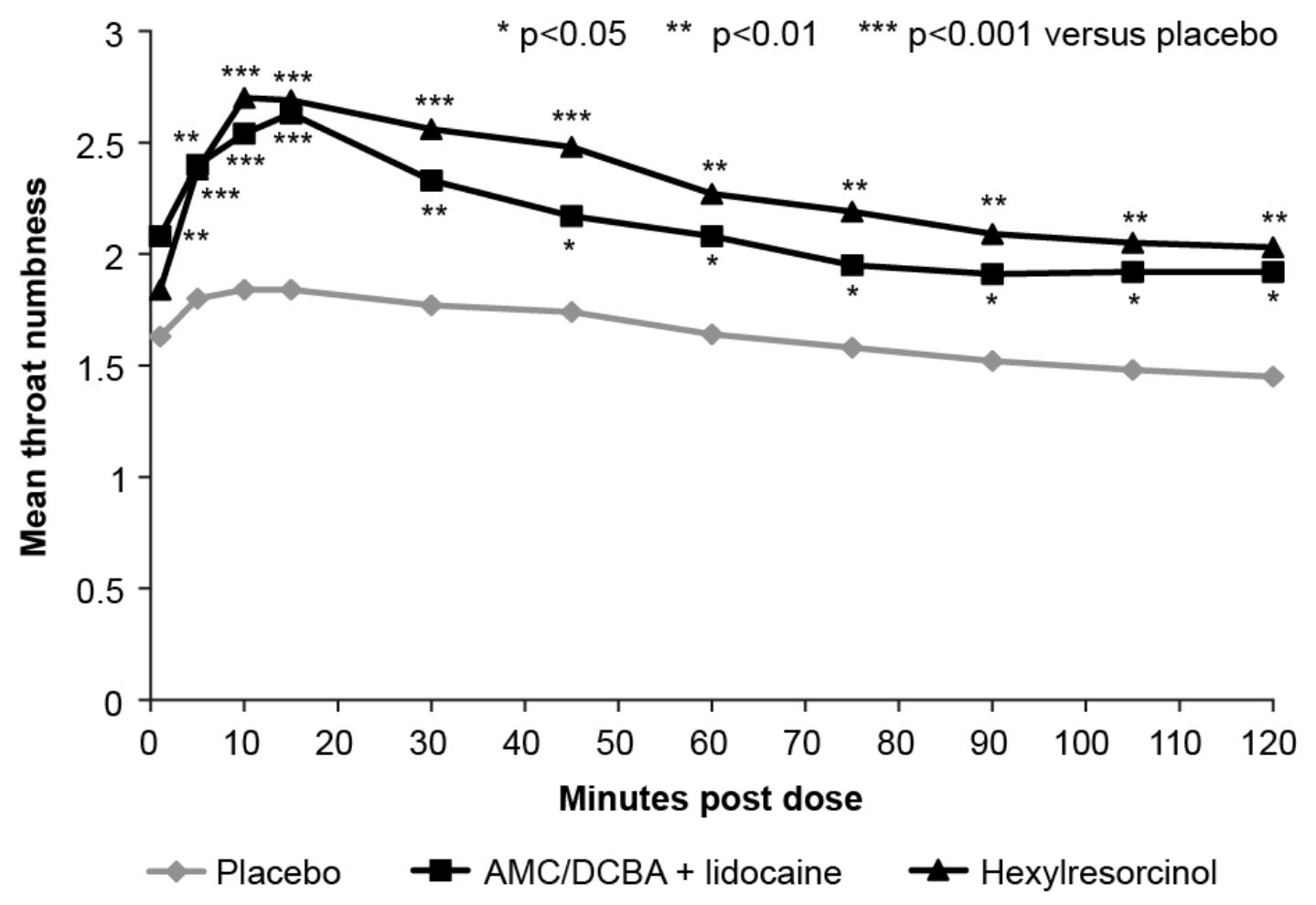


Figure 2 Continued.....

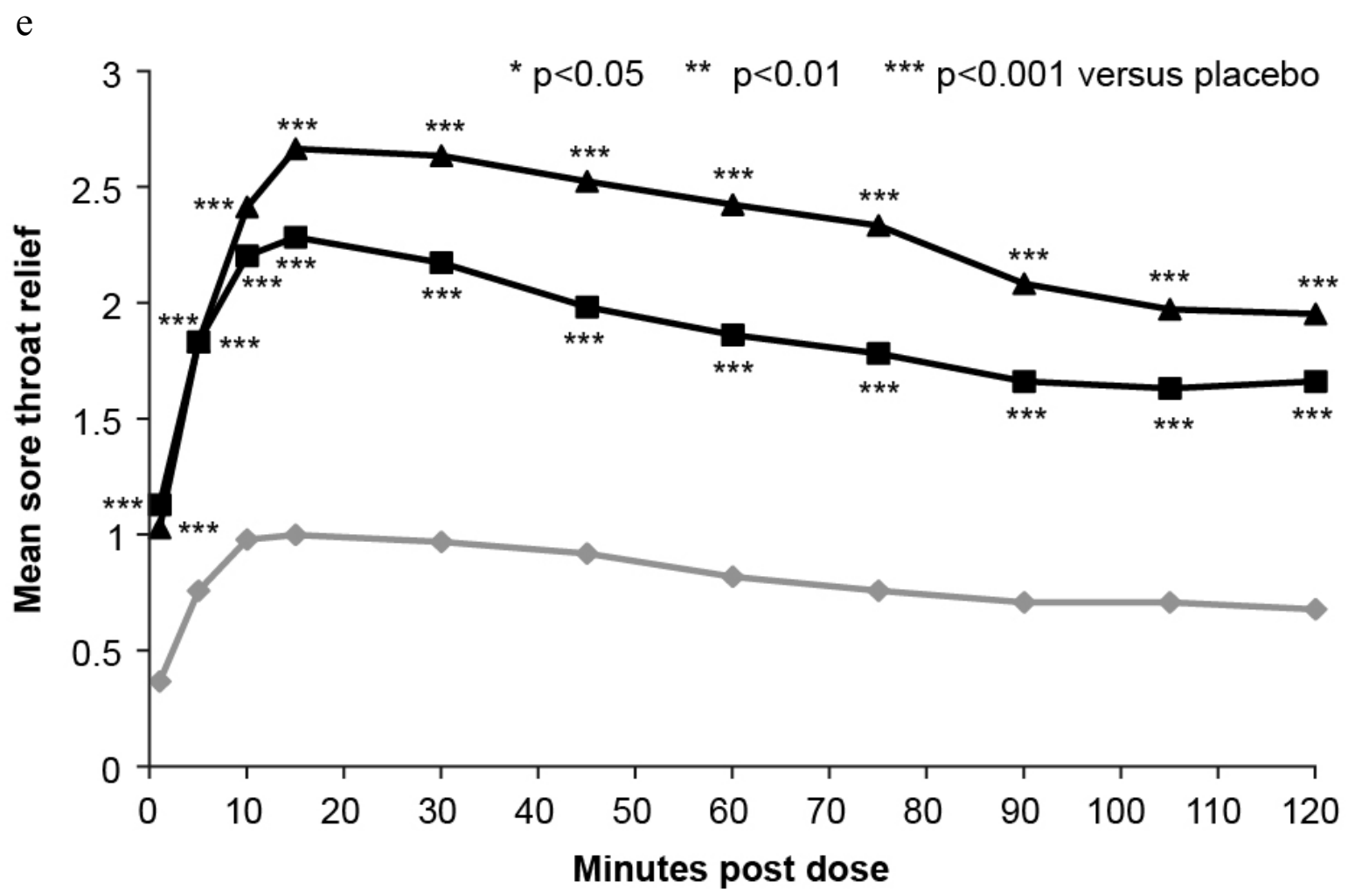

$\leadsto$ Placebo $\rightarrow$ AMC/DCBA + lidocaine $\rightarrow$ Hexylresorcinol

Figure 2. Change from Baseline in Throat Soreness (a), Difficulty Swallowing (b), and Swollen Throat (c), and Mean Throat Numbness (d) and Sore Throat Relief (e) for Hexylresorcinol and AMC/DCBA + Lidocaine Lozenges Versus Placebo. The coefficient of variation $(\min -\max )$ for each endpoint and treatment (AMC/DCBA + lidocaine lozenge, Hexylresorcinol lozenge and placebo lozenge, respectively) were as follows: throat soreness; -214.5--96.1), -183.9-73.0 and -340.4--139.7; difficulty swallowing; -170.5--100.6, -149.9--75.7 and -281.0--135.6; swollen throat; -466.3-130.2, -204.9--95.4 and -636.3--201.5; mean throat numbness; 39.3-63.1, 33.7-66.7 and 45.4-56.9 and sore throat relief; 58.7-114.8, 52.5-102.2 and 109.1-190.

The numb feeling lasted up to 2 hours. Furthermore, the hexylresorcinol lozenge had a long duration of action, with sore throat relief scores and mean change from baseline in throat soreness remaining significantly better than placebo at 2 hours after the dose.

For the AMC/DCBA + lidocaine lozenge, superiority over placebo was demonstrated but statistical significance was not consistent across all endpoints. Variability in the results obtained with the various endpoints used in the study might be expected because the scales evaluate different qualities of sore throat pain (21). This formulation had a rapid onset of action that was evident from 1-10 minutes after the dose.

The maximal effect was evident on most outcomes at 15 minutes, with onset of moderate pain relief at 30 minutes. Numbness was reported from 1 minute post dose, and was maximal at 15 minutes. This rapid anaesthestic action of lidocaine is well known and has been reported previously following delivery in a lozenge (11). The AMC/DCBA + lidocaine lozenge also had a long duration of action, with sore throat relief scores remaining significantly better than placebo at 2 hours after the dose. 
Table 4. Practitioner's Clinical Assessment of the Study Medication as a Treatment of Sore Throat and Patient's Global Evaluation of the Study Medication as a Treatment of Sore Throat at 2 hours Post Dose

\begin{tabular}{|c|c|c|c|c|c|}
\hline & $\begin{array}{c}\text { AMC/DCBA } \\
+ \text { lidocaine } \\
\text { lozenge } \\
\end{array}$ & $\begin{array}{l}\text { Hexyl- } \\
\text { resorcinol } \\
\text { lozenge } \\
\end{array}$ & Placebo & $\begin{array}{c}\mathrm{AMC} / \mathrm{DCBA}+ \\
\text { lidocaine lozenge vs } \\
\text { placebo }\end{array}$ & $\begin{array}{l}\text { Hexyl-resorcinol } \\
\text { lozenge vs placebo }\end{array}$ \\
\hline \multicolumn{6}{|l|}{ Patient } \\
\hline $\mathrm{N}$ & 64 & 64 & 62 & & \\
\hline 1 Poor & $12(18.8 \%)$ & $8(12.5 \%)$ & $39(62.9 \%)$ & & \\
\hline 2 Fair & $21(32.8 \%)$ & $21(32.8 \%)$ & $10(16.1 \%)$ & & \\
\hline 3 Good & $19(29.7 \%)$ & $16(25.0 \%)$ & $7(11.3 \%)$ & & \\
\hline 4 Very good & $10(15.6 \%)$ & $11(17.2 \%)$ & $6(9.7 \%)$ & & \\
\hline 5 Excellent & $2(3.1 \%)$ & $8(12.5 \%)$ & $0(0.0 \%)$ & & \\
\hline Mean \pm SD & $2.52 \pm 1.07$ & $2.84 \pm 1.22$ & $1.68 \pm 1.02$ & & \\
\hline LS mean ${ }^{a}$ & 2.47 & 2.81 & 1.64 & & \\
\hline Difference in LS mean ${ }^{\mathrm{b}}$ & & & & $\begin{array}{c}0.84 \\
(95 \% \text { CI } 0.45 \text { to } 1.23) \\
\text { p }<0.0001\end{array}$ & $\begin{array}{c}1.17 \\
(95 \% \text { CI } 0.78 \text { to } 1.56) \\
\mathrm{p}<0.0001\end{array}$ \\
\hline \multicolumn{6}{|l|}{ Clinician } \\
\hline $\mathrm{N}$ & 64 & 64 & 62 & & \\
\hline 1 Poor & $12(18.8 \%)$ & $11(17.2 \%)$ & $40(64.5 \%)$ & & \\
\hline 2 Fair & $20(31.3 \%)$ & $18(28.1 \%)$ & $12(19.4 \%)$ & & \\
\hline 3 Good & $20(31.3 \%)$ & $21(32.8 \%)$ & $5(8.1 \%)$ & & \\
\hline 4 Very good & $11(17.2 \%)$ & $9(14.1 \%)$ & $4(6.5 \%)$ & & \\
\hline 5 Excellent & $1(1.6 \%)$ & $5(7.8 \%)$ & $1(1.6 \%)$ & & \\
\hline Mean \pm SD & $2.52 \pm 1.04$ & $2.67 \pm 1.16$ & $1.61 \pm 1.00$ & & \\
\hline LS mean $^{\mathrm{a}}$ & 2.50 & 2.65 & 1.59 & & \\
\hline Difference in LS mean ${ }^{\mathrm{b}}$ & & & & $\begin{array}{c}0.91 \\
(95 \% \text { CI } 0.53 \text { to } 1.28) \\
p<0.0001\end{array}$ & $\begin{array}{c}1.06 \\
(95 \% \text { CI } 0.69 \text { to } 1.44) \\
\mathrm{p}<0.0001\end{array}$ \\
\hline
\end{tabular}

The data did not reach statistical significance on the primary endpoint with the AMC/DCBA + lidocaine lozenge. The sample size calculation used for the current trial was based on data obtained with an AMC/DCBA lozenge formulation. Variability in the current trial was higher than expected (SD 2.24 versus 1.78 expected), and the difference seen between the AMC/DCBA + lidocaine lozenge and placebo (0.75) was less than that used in the sample size calculation (1.21). Consequently, the sample size calculation was not predictive and the sample size was too small. To reach a p-value of 0.05 the study would have needed eight more subjects per group.

The minimal previous clinical experience was a major factor in the selection of the primary endpoint; based on the current findings, this was probably not the most representative candidate of lozenge efficacy. As anaesthetic action is a major factor in efficacy of treatment for sore throat and it is clear that the numbing effect for the AMC/DCBA + lidocaine lozenge formulation decreases over 2 hours (more so than with the hexylresorcinol formulation), a primary endpoint selected at the end of the period of drug action (2 hours) should not be the only factor considered when evaluating overall efficacy. It is also conceivable that the ordinal throat soreness scale represented a less defined descriptor of pain than the more overt categorical sore throat relief scale.

The scales for throat soreness and sore throat relief are accepted and validated analgesic assessment methodologies and have been used in previous clinical studies (16-21). Because patients with sore throat also frequently complain of other qualities of pain (in particular, difficulty swallowing and a swollen sensation in the throat), these qualities of pain were measured using the difficulty swallowing scale $(20,21)$ and the swollen throat scale $(20,21)$. As well as assessing different qualities of sore throat, these scales are 
constructed differently, with some being categorical and some continuous (VAS). These factors are probably responsible for some of the differences between outcomes, for example, timing of onset of analgesia versus numbness.

The current data are considered robust, the trial being randomised, placebo-controlled, and double-blind. However, limitations of the study include the relatively small sample size which may have limited the statistical power. This was likely a large factor in the failure of the AMC/DCBA + lidocaine lozenge data to reach statistical significance on the primary endpoint. It may also explain some of the inconsistencies seen, for example the apparent gap in the efficacy of the AMC/DCBA + lidocaine lozenge at 60 minutes on the swollen throat scale. Future research warrants the use of a larger sample size and careful selection of the most appropriate endpoints.

In conclusion, both the AMC/DCBA + lidocaine and hexylresorcinol lozenges provided rapid and effective sore throat relief in patients with URTI.

\section{ACKNOWLEDGEMENTS}

Our thanks go to the site coordinators. Medical writing assistance was provided by Elements Communications Ltd, supported by Reckitt Benckiser Healthcare International Ltd.

\section{FINANCIAL DISCLOSURE}

This study was funded by Reckitt Benckiser Healthcare International Ltd.

\section{REFERENCES}

1. Bisno AL, Gerber MA, Gwaltney JM Jr, Kaplan EL, Schwartz RH. Diagnosis and management of group A streptococcal pharyngitis: a practice guideline. Infectious Diseases Society of America. Clin Infect Dis, 1997; 25:574-583.

2. Vincent MT, Celestin N, Hussain AN. Pharyngitis. Am Fam Physician, 2004; 69(6):1465-1470.

3. Middleton DB. Pharyngitis. Prim Care, 1996; 23(4):719-739.

4. Gerber MA. Diagnosis and treatment of pharyngitis in children. Pediatr Clin North Am, 2005; 52(3):729-747, vi.

5. Bisno AL. Acute pharyngitis. N Engl J Med, 2001; 344(3):205-211.

6. NICE Clinical Guideline 69. Respiratory tract infections - antibiotic prescribing. National Institute for Health and Clinical Excellence, July 2008.
7. Limb M, Connor A, Pickford M, Church A, Mamman R, Reader S, Shephard A, Aspley S, Goulder MA. Scintigraphy can be used to compare delivery of sore throat formulations. Int $\mathrm{J}$ Clin Pract, 2009; 63(4):606-612.

8. McNally D, Simpson M, Morris C, Shephard A, Goulder M. Rapid relief of acute sore throat with AMC/DCBA throat lozenges: randomised controlled trial. Int J Clin Pract, 2010; 64(2): 194207.

9. Wade AG, Morris C, Shephard A, Crawford GM, Goulder MA. A multicentre, randomised, doubleblind, single-dose study assessing the efficacy of AMC/DCBA Warm lozenge or AMC/DCBA Cool lozenge in the relief of acute sore throat. BMC Fam Pract, 2011; 12:6.

10. Ebneshahidi A and Mohseni M. Strepsils ${ }^{\circledR}$ tablets reduce sore throat and hoarseness after tracheal intubation. Anesth Analg, 2010; 111(4):892-894.

11. Wonnemann M, Helm I, Stauss-Grabo M, RöttgerLuer P, Tran CT, Canenbley R, Donath F, Nowak $\mathrm{H}$, Schug BS, Blume HH. Lidocaine $8 \mathrm{mg}$ sore throat lozenges in the treatment of acute pharyngitis. A new therapeutic option investigated in comparison to placebo treatment. Arzneimittelforschung, 2007; 57(11):689-697.

12. Kriukov AI, Magomedov MM, Uzdennikov AA, Podmazov AV, Sedinkin AA, Maksimenko BIa. [Strepsils plus in the treatment of pharyngeal inflammation]. Vestn Otorinolaringol, 1999; (1):51-52.

13. Buchholz V, Leuwer M, Ahrens J, Foadi N, Krampfl K, Haeseler G. Topical antiseptics for the treatment of sore throat block voltage-gated neuronal sodium channels in a local anaestheticlike manner. Naunyn Schmiedebergs Arch Pharmacol, 2009; 380(2):161-168.

14. McNally D and Scheiner M. Acute sore throat, Module 1551. Available at: http://www.chemistanddruggist.co.uk/maincontent/-/article_display_list/4406146/updatemodule-1551-acute-sore-throat [Accessed on 07 November 2011].

15. Burnett I, Schachtel B, Sanner K, Bey M, Grattan T, Littlejohn S. Onset of analgesia of a paracetamol tablet containing sodium bicarbonate: A double-blind, placebo-controlled study in adult patients with acute sore throat. Clin Ther, 2006; 28(9):1273-1278.

16. Schachtel BP, Fillingim JM, Thoden WR, Lane AC, Baybutt RI. Sore throat pain in the evaluation of mild analgesics. Clin Pharmacol Ther, 1988; 44(6):704-711.

17. Watson N, Nimmo WS, Christian J, Charlesworth A, Speight J, Miller K. Relief of sore throat with the anti-inflammatory throat lozenge flurbiprofen 8.75 mg: a randomised, double-blind, placebocontrolled study of efficacy and safety. Int J Clin Pract, 2000; 54(8):490-496.

18. Benrimoj SI, Langford JH, Christian J, Charlesworth A, Steans A. Efficacy and 
tolerability of the anti-inflammatory throat lozenge flurbiprofen $8.75 \mathrm{mg}$ in the treatment of sore throat: a randomised, double-blind, placebocontrolled study. Clin Drug Invest, 2001; 21(3):183-193.

19. Blagden M, Christian J, Miller K, Charlesworth A. Multidose flurbiprofen $8.75 \mathrm{mg}$ lozenges in the treatment of sore throat: a randomised, doubleblind, placebo-controlled study in UK general practice centres. Int J Clin Pract, 2002; 56(2):95100.

20. Schachtel BP., Sore throat pain. In: Max MB, Portnoy RK, and Laska EM (eds). The Design of Analgesic Clinical Trials (Advances in pain research and therapy; Vol. 18). Raven Press, New York, pp 393-406, 1991.

21. Schachtel BP, McCormick CG, Giefer EE. Patient-Reported Outcomes (PROs) in the Pharyngitis Pain Model. Abstract PM035, 13th World Congress on Pain, Montreal 2010.

22. Wade AG., A randomized double-blind, parallel group, placebo-controlled, multiple-dose study of the efficacy of Strepsils lozenges in the relief of acute sore throat. In: Oxford JS (ed). Developments in acute sore throat relief - efficacy and sensorial benefits of medicated lozenges. Royal Society of Medicine Press Ltd, London, pp 22-32, 2008 [BH5013R]. 